\title{
Effects of exercise on L-carnitine and lipid metabolism in African catfish (Clarias gariepinus) fed different dietary L-carnitine and lipid levels
}

\author{
Rodrigo O. A. Ozorio ${ }^{1}$, Vincent J. T. Van Ginneken ${ }^{2}$, Rui J. B. Bessa ${ }^{3}$, Martin W. A. Verstegen ${ }^{4}$, \\ Johan A. J. Verreth ${ }^{5}$ and Elbertus A. Huisman ${ }^{5}$ \\ ${ }^{1}$ CIMAR/CIIMAR, Centro Interdisciplinar de Investigação Marinha e Ambiental, Universidade do Porto, Rua dos Bragas 289, \\ 4050-123 Porto, Portugal \\ ${ }^{2}$ Plant Research International, Agrosystems Research, PO Box 616, 6700 AP Wageningen, The Netherlands \\ ${ }^{3}$ CIISA, Faculdade de Medicina Veterinária, Universidade Técnica de Lisboa, Polo Universitário do Alto da Ajuda, 1300-477 \\ Lisbon, Portugal \\ ${ }^{4}$ Wageningen University and Research Centre, Animal Nutrition Group, NL-6709 PG Wageningen, The Netherlands \\ ${ }^{5}$ Wageningen University and Research Centre, Fish Culture and Fisheries Group, NL-6700 AH Wageningen, The Netherlands \\ (Received 14 July 2009 - Revised 20 October 2009 - Accepted 21 October 2009 - First published online 24 November 2009)
}

\begin{abstract}
African catfish (Clarias gariepinus) were fed four isonitrogenous diets (34\% crude protein), each containing one of two lipid (100 or $180 \mathrm{~g} / \mathrm{kg}$ ) and two L-carnitine (15 or $1000 \mathrm{mg} / \mathrm{kg}$ ) levels. After $81 \mathrm{~d}$ of feeding, thirty-two fish (body weight $32 \mathrm{~g}$ ) from each dietary group were randomly selected, sixteen fish were induced to a 3-h swim (speed of 1.5 body length (BL)/s), while the other sixteen fish were kept under resting condition. Fish fed $1000 \mathrm{mg}$ L-carnitine accumulated 3.5 and 5 times more L-carnitine in plasma and muscle, respectively, than fish fed the $15 \mathrm{mg}$ L-carnitine. Muscle L-carnitine content was significantly lower in exercised fish than in rested fish. High dietary lipid level (fish oil) led to an increase in muscle n-3 PUFA content and a decrease in SFA and MUFA content. In liver, the increase in dietary lipid level resulted in an increased levels of both $n-6$ and $n$-3 PUFA. L-carnitine supplementation significantly decreased $n-3$ PUFA content. Exercise decreased $n$ - 3 PUFA in both muscle and liver. Plasma lactate and lactate dehydrogenase, normally associated with increased glycolytic processes, were positively correlated with exercise and inversely correlated with dietary L-carnitine level. L-carnitine supplementation reduced significantly the RQ from $0 \cdot 72$ to 0.63 , and an interaction between dietary L-carnitine and lipid was observed $(P<0.03)$. Our results indicate that an increase in fatty acids $(\mathrm{FA})$ intake may promote FA oxidation, and both carnitine and exercise might influence the regulation of FA oxidation selectivity.
\end{abstract}

African catfish: L-carnitine metabolism: Lipid metabolism: Exercise

Fish are subjected to many changes in energy demand throughout their lifespan. As the lipid content of their diet increases so does the optimal dietary level of L-carnitine ${ }^{(1-3)}$, a multi-physiological, bioactive additive compound, synthesised from two essential amino acids, protein-bound lysine and methionine. It is not entirely understood whether animals require exogenous L-carnitine, and over the past 20 years, the scientific discussion regarding this subject continued with ambiguous arguments.

L-Carnitine plays several important physiological roles like shuttling the long-chain fatty acid (FA) across the inner mitochondrial membrane for ATP production ${ }^{(4,5)}$ and inhibition of superoxide radical formation ${ }^{(6)}$. According to Heo et al. ${ }^{(2,7)}$, L-carnitine biosynthesis might be sufficient to maintain growth of $20-\mathrm{kg}$ pigs during optimum husbandry conditions. Nevertheless, extra dietary L-carnitine is efficiently retained in body tissues, playing a role in nutrient utilisation and thus on growth performance and body composition. The rate of lipid oxidation is determined by the energy requirements of working muscles and by the availability of free L-carnitine delivery to muscle mitochondria ${ }^{(8,9)}$.

Since exogenous L-carnitine may influence energy utilisation efficiency, several studies using birds and mammals as animal models tested the effect of dietary L-carnitine supplementation on the exercise performance and on lipid metabolism ${ }^{(10-13)}$. Extra dietary L-carnitine has shown to improve, to a certain degree, the professional sport performances $^{(14)}$ and has improved exercise endurance in rats exposed to short and long-term exercise ${ }^{(15,16)}$. However, little is known about the effects of dietary L-carnitine supplements on the energy metabolism of exercised fish. Ozório et al. ${ }^{(17)}$ working with the African catfish fed high-fat level $(190 \mathrm{~g} / \mathrm{kg}$ diet $)$, showed that dietary L-carnitine supplementation alleviated fatigue during short-term exhaustive exercise by facilitating the recovery of muscle high-energy phosphates content (ATP and phosphocreatine). Ozório et al. ${ }^{(17)}$ suggested that muscle ATP recovery was stimulated from body lipids.

Abbreviation: FA, fatty acids.

*Corresponding author: Rodrigo O. A. Ozorio, fax +351 223390608, email rodrigo.ozorio@ciimar.up.pt 
Simultaneous respirometric measurements using the differential concentration of $\mathrm{O}_{2}, \mathrm{CO}_{2}$ and total ammonia nitrogen is useful tool to accurately determine substrate preferences under forced swimming activity ${ }^{(18-20)}$. The oxygen consumption of swimming fish will increase considerably above its resting level. The level of the RQ $\left(\mathrm{RQ}=\mathrm{CO}_{2 \text { produced }} /\right.$ $\mathrm{O}_{2 \text { consumed }}$ ) indicates the type of fuel being utilised under different metabolic conditions. The RQ for the catabolism of carbohydrates, lipids, and protein are 1.0, 0.7 and about 0.8 , respectively. Under resting conditions, the RQ of rainbow trout (Oncorhynchus mykiss), goldfish and African catfish range from 0.83 to $1 \cdot 0^{(21,22)}$. When African catfish is fed extra L-carnitine, RQ and total ammonia nitrogen tend to decrease, indicating better use of lipids and a decrease of protein catabolism, the so-called protein sparing action.

The aim of the present study was to study the combined effect of dietary L-carnitine and lipid levels on lipid metabolism in the African catfish (Clarias gariepinus) exposed to forced swimming exercise, a condition common both in nature and in intensive aquaculture systems.

\section{Materials and methods}

This experiment was approved by the Ethical Committee Judging Animal Experiments (DEC) of Wageningen University.

\section{Fish and facilities}

The experiment was conducted in the experimental facility, 'De Haar vissen' of the Wageningen University, The Netherlands. The African catfish (C. gariepinus) had a starting weight of 8.4 (SEM 0.3) g. The experimental animals were siblings with an identical nutritional history. The fish were kept in sixteen glass aquaria of 70 litres each, connected to a recirculation system. Water temperature $\left(25 \cdot 2 \pm 0 \cdot 6^{\circ} \mathrm{C}\right)$ and electric conductivity $(6.5 \pm 1.2 \mathrm{mS} / \mathrm{cm})$ were daily checked.

Dissolved oxygen (11.1 (SEM 2.0) mg/l), $\mathrm{pH}(7 \cdot 3$ (SEM 0.3)), $\mathrm{NH}_{4}^{+}\left(0.2\right.$ (SEM 0.2) mg/l), $\mathrm{NO}_{3}(137.5$ (SEM 78.9) $\mathrm{mg} / \mathrm{l})$ and $\mathrm{NO}_{2}(0.2$ (SEM $\left.0 \cdot 2) \mathrm{mg} / \mathrm{l}\right)$ were weekly checked.

\section{Experimental diet and design}

Four diets were selected to test two L-carnitine levels (15 or $1000 \mathrm{mg} / \mathrm{kg}$ ) and two lipid levels (100 or $180 \mathrm{~g} / \mathrm{kg}$; Table 1). Carniking (50\% L-carnitine, $35 \%$ silica and $15 \%$ water, Lonza) was used to increase the dietary carnitine level. All the diets were isoproteic (336-340 g crude protein $/ \mathrm{kg}$ diet) and had equal amounts of fishmeal. To obtain a basal diet with low L-carnitine level, fishmeal (main L-carnitine source ingredient) was kept at low level $(153 \mathrm{~g} / \mathrm{kg}$ diet $)$. Thus, to obtain isoproteic diets at $340 \mathrm{~g} / \mathrm{kg}$, gluten, feather meal and blood meal was adjusted accordingly. To keep the diets close to isoenergetic $(20 \mathrm{MJ} / \mathrm{kg})$ at different lipid levels, capelin oil was exchanged by starch and wheat. Fat levels were set at the out margins of the accepted inclusion range for African catfish $^{(23)}$. In addition, to enhance the effects of dietary L-carnitine supplementation, biosynthesis was set to a minimum by formulating diets with a low, but sufficient, lysine levels. The later was achieved by using a combination of wheat meal and maize gluten as primary protein sources.
Table 1. Ingredients, proximate composition and fatty acid (FA) content of diets containing different L-carnitine $(15$ or $1000 \mathrm{mg} / \mathrm{kg}$ ) and lipid $(100$ or $180 \mathrm{~g} / \mathrm{kg})$ levels*

\begin{tabular}{|c|c|c|c|c|}
\hline \multirow{2}{*}{$\begin{array}{l}\text { L-Carnitine } \\
\text { Lipid }\end{array}$} & \multicolumn{2}{|c|}{15} & \multicolumn{2}{|c|}{1000} \\
\hline & 100 & 180 & 100 & 180 \\
\hline \multicolumn{5}{|l|}{ Ingredients } \\
\hline Fishmeal & $153 \cdot 1$ & 152.9 & $153 \cdot 1$ & 152.9 \\
\hline Feather meal & $102 \cdot 0$ & - & $102 \cdot 0$ & - \\
\hline Gluten & 183.7 & 314.0 & 183.7 & 314.0 \\
\hline Wheat & 133.7 & 213.0 & 133.7 & 213.0 \\
\hline Pregelatinised maize starch & $255 \cdot 1$ & $86 \cdot 6$ & $255 \cdot 1$ & $86 \cdot 6$ \\
\hline Capelin oil & $56 \cdot 1$ & $122 \cdot 3$ & $56 \cdot 1$ & $122 \cdot 3$ \\
\hline Vitamin and mineral premix $\dagger$ & $5 \cdot 1$ & $5 \cdot 1$ & $5 \cdot 1$ & $5 \cdot 1$ \\
\hline Durabon binder & $25 \cdot 5$ & 24.5 & $25 \cdot 5$ & 24.5 \\
\hline Cellulose & 84.7 & $80 \cdot 6$ & 84.7 & $80 \cdot 6$ \\
\hline Carniking $(\mathrm{mg} / \mathrm{kg}) \ddagger$ & - & - & 1600 & 1600 \\
\hline \multicolumn{5}{|l|}{ Chemical composition } \\
\hline DM & $985 \cdot 1$ & 988.3 & $985 \cdot 1$ & $988 \cdot 3$ \\
\hline Crude protein & 335.9 & 339.2 & 335.9 & $339 \cdot 2$ \\
\hline Crude lipid & 106.9 & $185 \cdot 75$ & $106 \cdot 9$ & 185.75 \\
\hline Ash & $135 \cdot 1$ & 133.4 & $135 \cdot 1$ & 133.4 \\
\hline Carbohydrate§ & $326 \cdot 0$ & $240 \cdot 3$ & $326 \cdot 0$ & $240 \cdot 3$ \\
\hline Gross energy (MJ/kg) & $19 \cdot 2$ & $21 \cdot 6$ & $19 \cdot 4$ & 21.5 \\
\hline Lys & 11.4 & 11.5 & $11 \cdot 7$ & 11.4 \\
\hline Met & 3.24 & 2.56 & 5.58 & 2.87 \\
\hline L-Carnitine (mg/kg) & 14.3 & $17 \cdot 1$ & $972 \cdot 0$ & 1071.0 \\
\hline \multicolumn{5}{|l|}{$\mathrm{FA}(\mathrm{g} / \mathrm{kg})$} \\
\hline $14: 0$ & $3 \cdot 16$ & 5.93 & 3.19 & $6 \cdot 21$ \\
\hline $16: 0$ & $12 \cdot 21$ & 21.97 & $12 \cdot 31$ & 22.73 \\
\hline $18: 0$ & $2 \cdot 11$ & $3 \cdot 38$ & $2 \cdot 21$ & 3.50 \\
\hline $16: 1$ & 3.64 & 6.51 & 3.50 & 6.87 \\
\hline $18: 1$ & $13 \cdot 61$ & $24 \cdot 24$ & 14.08 & $25 \cdot 11$ \\
\hline $20: 1$ & $2 \cdot 30$ & 5.40 & $2 \cdot 34$ & 5.69 \\
\hline $22: 1$ & 3.55 & 8.48 & 3.65 & 8.69 \\
\hline $18: 2 n-6$ & 6.62 & 10.53 & 6.76 & 11.04 \\
\hline $18: 3 n-3$ & 0.78 & 1.48 & 0.76 & 1.56 \\
\hline $18: 4 n-3$ & 1.42 & 2.89 & 1.39 & 3.08 \\
\hline $20: 5 n-3$ & 5.56 & $10 \cdot 11$ & 5.57 & 10.69 \\
\hline $22: 5 n-3$ & 0.56 & 0.87 & 0.57 & 1.03 \\
\hline $22: 6 n-3$ & 5.34 & $10 \cdot 86$ & $5 \cdot 30$ & 11.39 \\
\hline$\Sigma$ SFA & $18 \cdot 12$ & 32.53 & $18 \cdot 37$ & 33.77 \\
\hline$\sum$ MUFA & 23.73 & 45.97 & $24 \cdot 23$ & 47.67 \\
\hline$\sum(n-6)$ & 7.50 & 12.03 & 7.54 & 12.64 \\
\hline$\sum(n-3)$ & 13.99 & $27 \cdot 00$ & 13.96 & 28.59 \\
\hline
\end{tabular}

* Values are expressed as $\mathrm{g} / \mathrm{kg}$ as fed basis, unless otherwise stated.

† Ingredient supplied per kilogram of feed. Vitamins: $774 \mathrm{mg}$ Vitamin A; $6.25 \mathrm{mg}$ vitamin D; $20000 \mathrm{mg}$ vitamin E. Minerals: $501 \mathrm{mg} \mathrm{CuSO}_{4} ; 15000 \mathrm{mg} \mathrm{ZnSO}$; $0.01 \mathrm{mg} \mathrm{MnSO}_{4} ; 500 \mathrm{CoSO}_{4} ; 500 \mathrm{mg} \mathrm{KI} ; 35 \mathrm{mg} \mathrm{Na}_{2} \mathrm{SeO}_{3}$.

$\ddagger 65 \%$ L-carnitine, $20 \%$ silica and $15 \%$ water. Provided by Lonza Group Ltd (Basel, Germany and Switzerland).

$\S$ Gelatinised starch plus glucose was determined according to Goelema et al. ${ }^{(83)}$.

The experiment was carried out according to a $2 \times 2$ factorial design with four replications each. Fish were acclimated for $7 \mathrm{~d}$ to the laboratory conditions before the feeding trial. During the acclimatisation period, fish were fed close to apparent satiation with the experimental diet containing $100 \mathrm{~g}$ lipid/kg diet and non-supplemented L-carnitine level $(14 \mathrm{mg} / \mathrm{kg})$. Thereafter, fish were randomly allocated to sixteen aquaria (forty-five fish/aquarium) and hand-fed twice a day with one of the four experimental diets for $81 \mathrm{~d}$. Feed consumption and dead fish were recorded on a daily basis.

After the feeding trial, thirty-two fish from each dietary group were randomly selected, sixteen of them were induced to a 3 -h swim (speed of $1.5 \mathrm{BL} / \mathrm{s}$ ) in a Blazka swimming tunnel, previously described by Van Ginneken ${ }^{(24)}$, and the 


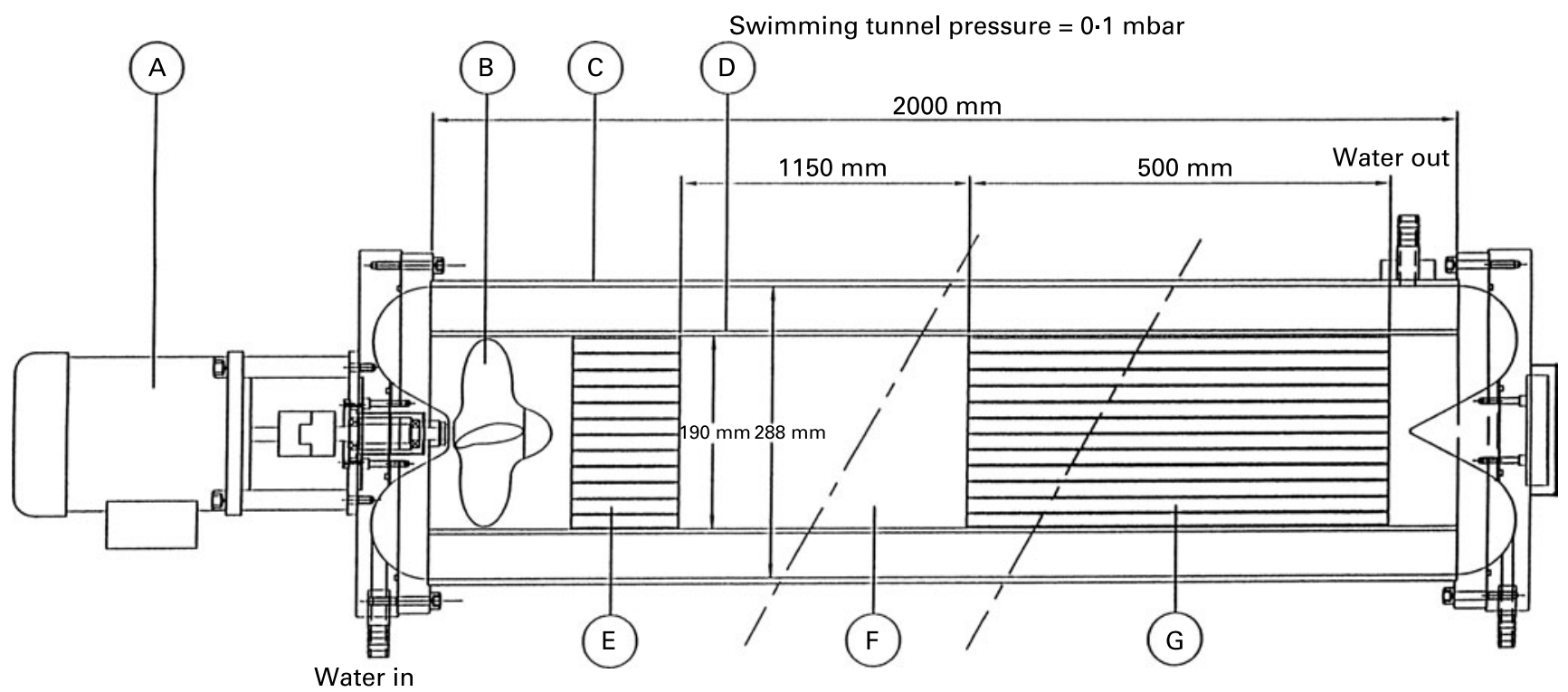

Fig. 1. Schematic drawing of a 2.0-m swim-tunnel. The tunnel consists of two concentric perspex tubes of $2 \mathrm{~m}$ and two PVC end-caps. A, electromotor; B, propeller; C, perspex outer swim-tunnel tube; D, perspex inner swim-tunnel tube; E, PVC end-streamer; F, animal compartment; G, PVC front streamer. The propeller pushes water into the outer ring and 'sucks it' out from the inner tube. The cross-section area of the inner tube and of the outer ring has the same surface area. This results in equal flow rates at both sides. The turbulent water is pushed through streamers that have internal diameters of about $10 \mathrm{~mm}$.

results were compared with the sixteen rested fish. The same handling procedures were performed on all fish to impose similar levels of stress on all animals before the swimming experiment.

The Blazka swimming tunnel (Fig. 1) had a length of $200 \mathrm{~cm}$ and consists of two tubes. The diameter of the outer and inner (the actual swimming tube) tubes were 28.8 and $19.0 \mathrm{~cm}$, respectively, with a total volume of 127 litres. The engine power was $400 \mathrm{~W}$ and the propeller consisted of three blades of $7.5 \mathrm{in}$. with a pitch of $7 \mathrm{in}$. The motor speed corresponding to $1.5 \mathrm{BL} / \mathrm{s}$ was calculated by the following equation: $Y=0.001307 \times X-0.01724$, elucidated by LaserDoppler techniques at the Hydraulics Laboratory TU (Delft, The Netherlands), where: $Y=$ water velocity in meter per second; $X=$ rpm of the engine of the Blazka swim tunnel. Here 0.001307 and 0.01724 are constants relating to the size of the tunnel and motor. This equation relates the number of rpm of the motor with the mean water velocity in the tunnel.

\section{Sampling, measurements and analytical procedure}

During the feeding trial, animals were weighed at the start and at the end of the experiment and total feed intake were determined for each aquarium at the end of the trial. Before every sampling, fish were fasted for 24-h, anaesthetised with tricaine methane sulphonate (Crescent Research Chemicals, Phoenix, AZ, USA), individually weighed and measured. The initial (twenty fish from the initial stock population) and final samples (thirty fish/treatment) of whole body were taken and stored at $-20^{\circ} \mathrm{C}$. During the final sampling, dorsal muscle sample and whole liver were withdrawn from nine fish per treatment, individually weighed and stored at $-80^{\circ} \mathrm{C}$ pending analyses.

Diet and muscle tissue were homogenised with a meat mincer, liver samples were pooled per aquarium before the homogenisation using an Ultra Torax. Blood $(0.5 \mathrm{ml} / \mathrm{fish})$ was collected from the caudal vein, centrifuged $(10 \mathrm{~min}$ at $\left.1500 \mathrm{~g}, 4^{\circ} \mathrm{C}\right)$ and plasma immediately stored at $-20^{\circ} \mathrm{C}$. After blood sampling, fish were sacrificed with an overdose of tricaine methane sulphonate buffered with $\mathrm{NaHCO}_{3}$ $(0 \cdot 3 \mathrm{~g}+0 \cdot 4 \mathrm{~g} / \mathrm{l}$ water $)$.

L-Carnitine was determined for diets, muscle and plasma according to Christiansen \& Bremer ${ }^{(25)}$. In brief, L-carnitine analyses were performed by radiometric detection of free and L-carnitine esters. The assay is based on the reaction of free L-carnitine with acetyl-CoA catalysed by L-carnitine acetyltransferase with the production of acetyl L-carnitine and coenzyme A. All the samples were deproteinated with perchloric acid, subsequently neutralised with $\mathrm{KOH}$, centrifuged and the supernatant was collected. L-carnitine was extracted by stepwise heating, ultrasonic treatment or extraction with various detergents. Additionally, alkaline hydrolysis was performed for the determination of $\mathrm{L}$-carnitine esters.

DM $\left(4 \mathrm{~h}\right.$ at $\left.103^{\circ} \mathrm{C}\right)$, ash $\left(6 \mathrm{~h}\right.$ at $\left.550^{\circ} \mathrm{C}\right)$, protein (macro-Kjeldahl $\mathrm{N} \times 6.25$ ), lipid (petroleum diethyl ether extraction using Soxlet) and energy (bomb calorimetry) analyses were determined for diets, muscle and liver samples.

FA concentrations of the diets, muscle and liver were determined as FA methyl esters according to Ozorio et al. ${ }^{(26)}$. Freeze-dried sample $(0.2 \mathrm{~g} / \mathrm{fish})$ and internal standard (1.99 mg Tricosanoic acid methyl ester $(23: 0) / \mathrm{ml}$ hexane) were saponified and esterified (dry bath, $22^{\circ} \mathrm{C}$ for $90 \mathrm{~min}$ ) with methanolic $\mathrm{KOH}$ and the methyl esters extracted into hexane (1 hexane:1 KOH). Supernatant hexane was then separated and taken for automatic injection $(2 \mu \mathrm{l} / \mathrm{sample})$ in a gas chromatograph (Varian 3300) with a cross linked $30 \mathrm{~m} \times 0.25 \mathrm{~mm}$ column silicate type, internal diameter of $0.53 \mathrm{~mm}$ and $\mathrm{H}_{2}$ as the carrier gas. The injection and detector temperatures were $280^{\circ} \mathrm{C}$ and peak identification and quantification was done by using the relative retention times of the reference FA methyl esters standards. 


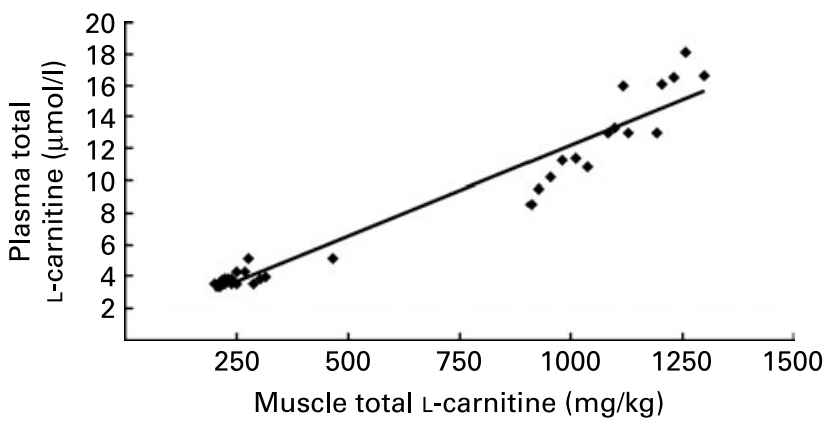

Fig. 2. Relationship between muscle and plasma total L-carnitine content (thirty-two observations). $y=0.0115 x+0.7474 ; R^{2} 0.94$.

Plasma analyses were carried out for total protein (colorimetric method, Roche Diagnostics GmbH, Mannheim, Germany), TAG (colorimetric method, Boehringer Mannheim Gmbh Diagnostica, Mannheim, Germany), cholesterol (cholesterol esterase colorimetric method, Boehringer Mannheim Gmbh Diagnostica), lactate dehydrogenase (kinetic determination of the LDH activity, Boehringer Mannheim Gmbh Diagnostica), lactate (enzymatic method, Roche Diagnostics $\mathrm{GmbH}$ ), urea (urease - glutamate dehydrogenase reaction, Boehringer Mannheim). All the above plasma assays were carried out using a Hitachi 717 Automatic Analyser from Boehringer Mannheim. Plasma glucose levels were determined with deproteinated samples by the GOD method (Boehringer Mannheim Gmbh Diagnostica) and NEFA were determined by NEFAC-Test kit (WAKO, GmbH, Germany) using ELISA reader at $420 \mathrm{~nm}$-wave length. Plasma cortisol levels were measured by RIA $^{(27)}$.

During the forced swimming trial, the RQ $\left(\mathrm{RQ}=\mathrm{CO}_{2 \text { produced }} /\right.$ $\left.\mathrm{O}_{2 \text { consumed }}\right)$ and nitrogen quotient $\left(\mathrm{NQ}=\mathrm{N}_{\text {produced }} / \mathrm{O}_{2 \text { consumed }}\right)$ were determined by using an automated auto analyser (Skalar B.V., Breda, The Netherlands) according to Ozório et al. ${ }^{(22)}$.

Table 3. Effects of dietary L-carnitine (15 or $1000 \mathrm{mg} / \mathrm{kg}$ ) and lipid (100 or $180 \mathrm{~g} / \mathrm{kg}$ ) levels on growth performance and feed utilisation of African catfish (Clarias gariepinus)

\begin{tabular}{|c|c|c|c|c|c|}
\hline \multirow{2}{*}{$\begin{array}{l}\text { Dietary L-carnitine } \\
\text { Dietary lipid }\end{array}$} & \multicolumn{2}{|c|}{15} & \multicolumn{2}{|c|}{1000} & \multirow[b]{2}{*}{ SEM } \\
\hline & 100 & 180 & 100 & 180 & \\
\hline Initial weight (g) & $8 \cdot 4$ & 8.4 & 8.5 & 8.4 & 0.1 \\
\hline Final weight (g) & $29 \cdot 2$ & $31 \cdot 7$ & $27 \cdot 1$ & $29 \cdot 6$ & 0.8 \\
\hline DGI $(\% \text { BW/d })^{*}$ & 1.27 & 1.38 & $1 \cdot 18$ & 1.29 & 0.03 \\
\hline VFI $(\%$ BW/d)† & $2 \cdot 40$ & $2 \cdot 31$ & $2 \cdot 11$ & 1.99 & 0.06 \\
\hline FCR $(g / g) \ddagger$ & $1 \cdot 79$ & 1.63 & 1.65 & 1.48 & 0.05 \\
\hline PER $(\mathrm{g} / \mathrm{g}) \S$ & 1.67 & 1.83 & 1.83 & 2.05 & 0.06 \\
\hline HSI (\% BW)\| & $4 \cdot 1$ & 4.4 & $3 \cdot 7$ & $4 \cdot 1$ & 0.1 \\
\hline Condition factorq & 0.6 & 0.6 & 0.6 & 0.6 & 0.0 \\
\hline Mortality (\%) & 13.9 & $9 \cdot 4$ & $11 \cdot 1$ & $11 \cdot 7$ & $3 \cdot 2$ \\
\hline
\end{tabular}

DGI, daily growth index; BW, body weight; VFI, voluntary feed intake; FCR, feed conversion ratio; PER, protein efficiency ratio; $\mathrm{HSI}$, hepatosomatic index. SEM, pooled standard error of mean: standard deviation $/ \sqrt{ } n$.

${ }^{*} \mathrm{DGI}=\left(\mathrm{FBW}^{1 / 3}-\mathrm{IBW}^{1 / 3} /(t)\right.$, where IBW, initial BW; FBW, final BW; $t$, trial duration. $\dagger \mathrm{VFI}=$ feed $[(100)$ (intake/average BW $(\mathrm{ABW}) /(t)]$, where $\mathrm{ABW}=(\mathrm{IBW}+\mathrm{FBW}) / 2$.

$\ddagger F C R=$ total dry feed intake $(\mathrm{g}) /$ total wet weight gain $(\mathrm{g})$.

$\S \mathrm{PER}=$ wet weight gain $(\mathrm{g}) / \mathrm{crude}$ protein intake $(\mathrm{g})$.

$\| \mathrm{HSI}=$ liver weight $\times 100 / \mathrm{BW}(\%)$

I $K=\left[(100)(\mathrm{FBW}, \mathrm{g}) /\left(\mathrm{Lt}^{3}, \mathrm{~cm}\right)\right]$, where $\mathrm{Lt}$ is the final standard length. 


\section{Statistical analysis}

The data were analysed as $3 \times 2$ factorial using a general linear model (Proc GLM, SAS, Cary, NC, USA) that included the effect of exercise (resting and swimming), dietary L-carnitine (15 and $1000 \mathrm{mg} / \mathrm{kg}$ diet) and dietary lipid (100 and $180 \mathrm{~g} / \mathrm{kg}$ diet) as main factors and their interactions. Differences between means were reported as significant if $P<0 \cdot 05$, using Bonferroni's multiple $t$ tests. Normality was tested using Shapiro-Wilk test. Homogeneity was checked using the absolute residuals according to Levene's test. Non-homogeneous data were arcsine transformed before further statistical analysis.

\section{Results}

Fish fed $1000 \mathrm{mg}$ L-carnitine accumulated 3.5-fold more L-carnitine in plasma than fish fed $15 \mathrm{mg} \mathrm{L}$-carnitine did (Table 2). Exercise had no detectable effect on total, free or esterified L-carnitine levels in plasma. Dietary L-carnitine significantly increased the L-carnitine levels in muscle by 5 -fold. There was a strong interaction between dietary L-carnitine and lipid levels, resulting in a steep increase in the free L-carnitine content with increasing dietary lipid level. Exercised fish retained significantly less free L-carnitine in muscle when compared with rested fish. Muscle total L-carnitine content was positively correlated to plasma total L-carnitine content (Fig. 2). The relationship between muscle and plasma L-carnitine content was fitted using a linear regression, described as $Y=0.0115 X+0.7474, R^{2} 0.94$. Plasma L-carnitine content also increased moderately when muscle L-carnitine accumulated from 200 to $500 \mathrm{mg} / \mathrm{kg}$, thereafter a sharp increase in plasma L-carnitine with increase in muscle L-carnitine was observed.

Depending upon the dietary treatment, fish grew from the initial $8.4 \mathrm{~g}$ to a mean final body weight of $27-32 \mathrm{~g}$ (Table 3). Growth rates varied between 1.18 and $1.38 \%$ body weight/d. The average mortality during the experiment ranged from 9 to $14 \%$ with no effect of diet. Dietary L-carnitine and lipid content had no significant effect on growth performance and feed utilisation.

Liver and muscle proximate composition was significantly affected as a result of experimental treatments (Table 4). DM content increased with the dietary lipid $(P<0.0003)$ and decreased with the dietary L-carnitine $(P<0 \cdot 009)$. Protein content was inversely related with the dietary lipid $(P<0.0001)$ and a three-way interaction was observed. Liver lipid content increased with increasing dietary lipid $(P<0.0003)$ and decreased with the L-carnitine levels $(P<0.0007)$. Exercise did not alter the lipid content in liver. Liver energy was significantly affected by all experimental treatments.

The effects of dietary composition and exercise were less evident in muscle proximate composition than in liver. Dietary lipid and L-carnitine levels did not affect muscle lipid content. Exercised fish tended to have higher DM content $(248-256 \mathrm{~g} / \mathrm{kg})$ than non-exercised fish $(235-246 \mathrm{~g} / \mathrm{kg})$. Exercised fish retained significantly more lipid than nonexercised fish, and an interaction between dietary lipid and exercise was observed.

FA content (mg/g lipids) in muscle (Table 5) and liver (Table 6) were influenced by exercise and dietary treatments. 
Table 5. Effects of dietary L-carnitine (15 or $1000 \mathrm{mg} / \mathrm{kg}$ ) and lipid (100 or $180 \mathrm{~g} / \mathrm{kg}$ ) levels and exercise on total fatty acid (FA) concentration (mg/g muscle and $\mathrm{mg} / \mathrm{g}$ lipids) and individual FA composition ( $\mathrm{mg} / \mathrm{g}$ lipids) of African catfish (Clarias gariepinus) muscle

\begin{tabular}{|c|c|c|c|c|c|c|c|c|c|c|}
\hline & \multicolumn{2}{|c|}{ Carnitine } & \multicolumn{2}{|c|}{ Lipid } & \multicolumn{2}{|c|}{ Exercise } & \multirow[b]{2}{*}{ SEM } & \multirow[b]{2}{*}{ C } & \multirow[b]{2}{*}{$\mathrm{L}$} & \multirow[b]{2}{*}{ E } \\
\hline & 15 & 1000 & 100 & 180 & Resting & Exercise & & & & \\
\hline \multicolumn{11}{|l|}{ FA } \\
\hline $\mathrm{FA}$ (mg/g muscle) & 174.0 & 184.0 & 178.0 & $179 \cdot 0$ & 181.0 & $177 \cdot 0$ & 0.0 & - & - & - \\
\hline FA (mg/g Lipids) & $718 \cdot 0$ & 785.0 & 768.0 & 734.0 & 773.0 & 729.0 & $15 \cdot 2$ & 0.004 & - & 0.050 \\
\hline $14: 0$ & 24.0 & $26 \cdot 4$ & 23.8 & $26 \cdot 6$ & 25.9 & 24.5 & 0.51 & 0.003 & $<0.001$ & 0.050 \\
\hline $16: 0$ & $172 \cdot 0$ & 191.0 & 197.0 & $167 \cdot 0$ & $187 \cdot 0$ & 176.0 & 3.7 & 0.001 & $<0.001$ & - \\
\hline $18: 0$ & 39.9 & $44 \cdot 8$ & $46 \cdot 6$ & $38 \cdot 1$ & 43.5 & $41 \cdot 2$ & 1.0 & 0.002 & $<0.001$ & - \\
\hline $16: 1 n-7$ & $36 \cdot 8$ & 41.0 & $42 \cdot 2$ & $35 \cdot 6$ & $39 \cdot 8$ & 38.0 & 0.8 & 0.001 & $<0.001$ & - \\
\hline $18: 1 n-9$ & $151 \cdot 0$ & $166 \cdot 0$ & 173.0 & $145 \cdot 0$ & $163 \cdot 0$ & $155 \cdot 0$ & 3.5 & 0.005 & $<0.001$ & - \\
\hline $18: 1 n-7$ & $16 \cdot 8$ & $18 \cdot 6$ & $18 \cdot 7$ & $16 \cdot 7$ & 18.4 & $17 \cdot 0$ & 0.4 & 0.002 & $<0.001$ & 0.013 \\
\hline $20: 1 n-9$ & $20 \cdot 1$ & $21 \cdot 6$ & $18 \cdot 6$ & $23 \cdot 1$ & $21 \cdot 6$ & $20 \cdot 1$ & 0.4 & 0.015 & $<0.001$ & 0.022 \\
\hline $22: 1 n-11$ & 15.4 & $17 \cdot 0$ & $13 \cdot 3$ & $19 \cdot 0$ & $16 \cdot 6$ & $15 \cdot 7$ & 0.4 & 0.007 & $<0.001$ & - \\
\hline $18: 2 n-6$ & $59 \cdot 3$ & $63 \cdot 7$ & $61 \cdot 6$ & 61.4 & 63.9 & 59.0 & 1.4 & 0.037 & - & 0.019 \\
\hline $18: 3 n-6$ & 1.36 & 1.34 & 1.25 & 1.44 & 1.37 & 1.33 & 0.04 & - & 0.002 & - \\
\hline $20: 2 n-6$ & $2 \cdot 64$ & 2.85 & $2 \cdot 61$ & 2.87 & 2.68 & 2.82 & 0.08 & - & 0.027 & - \\
\hline $20: 3 n-6$ & 2.93 & $3 \cdot 24$ & 3.69 & $2 \cdot 47$ & 3.21 & 2.96 & 0.09 & 0.021 & $<0.001$ & - \\
\hline $20: 4 n-6$ & $3 \cdot 21$ & 3.57 & 3.48 & $3 \cdot 29$ & 3.59 & $3 \cdot 19$ & 0.10 & 0.019 & - & 0.010 \\
\hline $22: 2 n-6$ & 3.92 & $4 \cdot 21$ & $4 \cdot 38$ & $3 \cdot 76$ & 3.37 & 4.77 & 0.25 & - & - & $<0.001$ \\
\hline $18: 3 n-3$ & $6 \cdot 10$ & 6.58 & 5.75 & 6.92 & 6.54 & $6 \cdot 13$ & 0.14 & 0.018 & $<0.001$ & 0.040 \\
\hline $18: 4 n-3$ & 8.47 & $8 \cdot 77$ & $7 \cdot 21$ & $10 \cdot 0$ & 8.89 & 8.34 & 0.21 & - & $<0.001$ & - \\
\hline $20: 3 n-3$ & 0.69 & 0.74 & 0.60 & 0.82 & 0.73 & 0.70 & 0.03 & - & $<0.001$ & - \\
\hline $20: 4 n-3$ & 3.49 & 3.86 & $3 \cdot 17$ & $4 \cdot 19$ & 3.78 & 3.57 & 0.09 & 0.011 & $<0.001$ & - \\
\hline $20: 5 n-3$ & $30 \cdot 3$ & $31 \cdot 1$ & $27 \cdot 6$ & 33.8 & $32 \cdot 1$ & 29.4 & 0.6 & - & $<0.001$ & 0.006 \\
\hline $22: 5 n-3$ & 8.38 & $9 \cdot 11$ & 8.38 & $9 \cdot 10$ & 9.00 & 8.47 & 0.18 & 0.007 & 0.008 & 0.043 \\
\hline $22: 6 n-3$ & $74 \cdot 1$ & 79.9 & $69 \cdot 6$ & 84.4 & $80 \cdot 1$ & $73 \cdot 8$ & 1.6 & 0.018 & $<0.001$ & 0.011 \\
\hline Total SFA & $242 \cdot 0$ & 269.0 & 273.0 & 237.0 & $263 \cdot 0$ & 248.0 & $5 \cdot 2$ & 0.001 & $<0.001$ & - \\
\hline Total MUFA & 252 & 278 & 277 & 253 & 272 & 258 & 5.5 & 0.004 & 0.004 & - \\
\hline$n-6$ & 73.4 & 78.9 & 77.0 & $75 \cdot 3$ & 78.2 & $74 \cdot 1$ & $1 \cdot 70$ & 0.032 & - & - \\
\hline$n-3$ & 131 & 140 & 122 & 149 & 141 & 130 & $2 \cdot 7$ & 0.037 & $<0.001$ & 0.010 \\
\hline Total PUFA & 205 & 219 & 199 & 225 & 219 & 204 & 4.4 & 0.033 & $<0.001$ & 0.025 \\
\hline
\end{tabular}

SEM, pooled standard error of mean: standard deviation $/ \sqrt{ } n ; n-3$ and $n-6$, total FA of $(n-3)$ or $(n-6)$ series; L-C, L-carnitine; L, lipid; E, exercise; -, NS.

In muscle, L-carnitine supplementation increased the content of most FA, whereas high dietary lipid level increased the $n-3$ PUFA and decreased the SFA and MUFA. The effect of exercise on muscle FA content was less pronounced but exercise decreased $n$-3 PUFA and tended $(P<0 \cdot 06)$ to decrease SFA. None of the interactions between main factors were significant.

In liver, L-carnitine supplementation decreased the n-3 PUFA and tended $(P<0 \cdot 07)$ to increase MUFA, whereas high dietary lipid level increased the $n$-3 PUFA and decreased the SFA and MUFA. However, a significant interaction between dietary levels of L-carnitine and lipids were observed for most of the $n-3$ PUFA, and also $18: 2 n-6$ and $22: 1 n-11$. All these FA increased with the level of lipid in the diet, but the increase was higher in fish fed non-supplemented Lcarnitine diet (Fig. 3). Exercised fish had lower n-3 PUFA levels in liver than non-exercised fish.

Table 7 shows the means of selected plasma parameters of fish fed different experimental diets, with or without swimming exercise. Dietary L-carnitine combined with exercise evoked significant changes on several plasma metabolites, such as cholesterol, lactate, lactate dehydrogenase, NEFA and cortisol. Cholesterol levels increased with increasing dietary L-carnitine. Plasma lactate decreased with increasing dietary L-carnitine. Plasma lactate and lactate dehydrogenase showed a significant increase during exercise. Plasma cortisol was significantly lower in the resting fish $(63 \mu \mathrm{g} / \mathrm{l})$ when compared with exercised fish $(166 \mu \mathrm{g} / \mathrm{l})$. There were interactions between dietary L-carnitine, lipid and exercise
$(P<0.02)$ on plasma cortisol and NEFA levels. Plasma protein and glucose levels were not affected by dietary treatments or exercise also L-carnitine and exercise tended to decrease plasma urea. Plasma TAG were slightly higher in fish fed $1000 \mathrm{mg}$ L-carnitine and tended to decrease with exercise.

The effects of dietary L-carnitine and lipid supplements on gas exchange, respiratory and nitrogen quotients in exercised African catfish is depicted in Table 8. During exercise, the $\mathrm{O}_{2}$ uptake tended to increase with dietary L-carnitine supplementation. The changes in $\mathrm{O}_{2}$ uptake led to a RQ ranging from 0.62 to 0.72 . Dietary L-carnitine supplementation significantly reduced the RQ from 0.72 to 0.63 , and an interaction between dietary L-carnitine and lipid was observed $(P<0.03)$.

Nitrogen $\left(\mathrm{N}-\mathrm{NH}_{4}^{+}\right)$excretion was significantly influenced by the dietary L-carnitine and lipid level $(P<0 \cdot 01)$. In groups supplemented with $1000 \mathrm{mg}$ L-carnitine, nitrogen excretion was significantly reduced when fed $180 \mathrm{~g}$ lipid $/ \mathrm{kg}$ diet $(0.93$ and $1.06 \mathrm{mmol} / \mathrm{kg}$ per h) compared with fish fed $100 \mathrm{~g}$ lipid ( 1.03 and $1.63 \mathrm{mmol} / \mathrm{kg}$ per $\mathrm{h})$, and an interaction between dietary L-carnitine and lipid levels was observed $(P<0.05)$. The nitrogen quotient ranged from 0.07 to 0.12 and was not affected by the dietary treatment.

\section{Discussion}

L-Carnitine is available from endogenous biosynthesis and from dietary sources. The body distribution of L-carnitine is 
Table 6. Effects of dietary L-carnitine (15 or $1000 \mathrm{mg} / \mathrm{kg}$ ) and lipid (100 or $180 \mathrm{~g} / \mathrm{kg}$ ) levels and exercise on total fatty acid (FA) concentration (mg/g tissue and $\mathrm{mg} / \mathrm{g}$ lipids) and individual FA composition (mg/g lipids) of African catfish (Clarias gariepinus) liver

\begin{tabular}{|c|c|c|c|c|c|c|c|c|c|c|}
\hline & \multicolumn{2}{|c|}{ Carnitine } & \multicolumn{2}{|c|}{ Lipid } & \multicolumn{2}{|c|}{ Exercise } & \multirow[b]{2}{*}{ SEM } & \multirow[b]{2}{*}{$\mathrm{C}$} & \multirow[b]{2}{*}{$\mathrm{L}$} & \multirow[b]{2}{*}{ E } \\
\hline & 15 & 1000 & 100 & 180 & Resting & Exercise & & & & \\
\hline \multicolumn{11}{|l|}{ FA } \\
\hline FA (mg/g liver) & 448 & 438 & 418 & 467 & 447 & 439 & 8 & - & $<0.001$ & - \\
\hline FA (mg/g lipids) & 611 & 643 & 609 & 645 & 635 & 619 & $14 \cdot 1$ & - & - & - \\
\hline $14: 0$ & $12 \cdot 1$ & $12 \cdot 2$ & $10 \cdot 4$ & $13 \cdot 8$ & $12 \cdot 4$ & 11.9 & 0.269 & - & $<0.001$ & - \\
\hline $16: 0$ & 188 & 206 & 202 & 192 & 200 & 194 & 4.5 & 0.008 & - & - \\
\hline $18: 0$ & 41.7 & 44.9 & $44 \cdot 3$ & $42 \cdot 3$ & 43.0 & 43.6 & 1.50 & - & - & - \\
\hline $16: 1 n-7$ & 41.7 & $46 \cdot 0$ & $48 \cdot 2$ & 39.6 & $44 \cdot 8$ & 43.0 & 1.09 & 0.008 & $<0.001$ & - \\
\hline $18: 1 n-9$ & 186 & 199 & 202 & 183 & 193 & 192 & $5 \cdot 62$ & - & 0.027 & - \\
\hline $18: 1 n-7$ & $14 \cdot 3$ & $16 \cdot 1$ & $15 \cdot 5$ & $15 \cdot 0$ & $15 \cdot 4$ & $15 \cdot 1$ & 0.30 & $<0.001$ & 0.252 & - \\
\hline $20: 1 n-9$ & 13.6 & $14 \cdot 1$ & 11.9 & $15 \cdot 9$ & 13.9 & $13 \cdot 8$ & 0.31 & - & $<0.001$ & - \\
\hline $22: 1 n-11$ & 2.39 & $2 \cdot 25$ & 1.27 & $3 \cdot 37$ & 2.37 & $2 \cdot 26$ & 0.046 & 0.039 & $<0.001$ & - \\
\hline $18: 2 n-6$ & $23 \cdot 8$ & $22 \cdot 4$ & $15 \cdot 9$ & $30 \cdot 3$ & $23 \cdot 7$ & $22 \cdot 5$ & 0.66 & - & $<0.001$ & - \\
\hline $18: 3 n-6$ & 1.05 & 0.96 & 0.76 & 1.25 & 1.04 & 0.97 & 0.035 & 0.054 & $<0.001$ & - \\
\hline $20: 2 n-6$ & 3.05 & 3.04 & 2.40 & 3.68 & 3.05 & 3.03 & 0.055 & - & $<0.001$ & - \\
\hline $20: 3 n-6$ & 4.66 & 4.70 & $4 \cdot 11$ & $5 \cdot 26$ & 4.81 & 4.55 & $0 \cdot 101$ & - & $<0.001$ & - \\
\hline $20: 4 n-6$ & 1.60 & 1.58 & 1.41 & $1 \cdot 76$ & 1.71 & 1.46 & 0.059 & - & $<0.001$ & 0.006 \\
\hline $22: 2 n-6$ & 7.47 & 7.53 & $8 \cdot 13$ & $6 \cdot 89$ & 6.85 & $8 \cdot 16$ & 0.485 & - & - & - \\
\hline $18: 3 n-3$ & 1.70 & 1.46 & 0.75 & $2 \cdot 40$ & 1.64 & 1.52 & 0.057 & 0.005 & $<0.001$ & - \\
\hline $18: 4 n-3$ & 0.66 & 0.52 & 0.31 & 0.87 & 0.64 & 0.54 & 0.032 & 0.005 & $<0.001$ & 0.028 \\
\hline $20: 3 n-3$ & 0.53 & 0.58 & 0.35 & 0.75 & 0.57 & 0.53 & 0.030 & - & $<0.001$ & - \\
\hline $20: 4 n-3$ & 1.88 & 1.52 & 0.65 & $2 \cdot 75$ & 1.81 & 1.59 & 0.072 & 0.001 & $<0.001$ & 0.034 \\
\hline $20: 5 n-3$ & $7 \cdot 17$ & $5 \cdot 30$ & 2.98 & 9.49 & $6 \cdot 61$ & $5 \cdot 87$ & 0.198 & $<0.001$ & $<0.001$ & 0.014 \\
\hline $22: 5 n-3$ & $5 \cdot 75$ & 4.49 & $2 \cdot 65$ & 7.59 & $5 \cdot 28$ & 4.96 & 0.154 & $<0.001$ & $<0.001$ & - \\
\hline $22: 6 n-3$ & $31 \cdot 2$ & $28 \cdot 1$ & $17 \cdot 1$ & $42 \cdot 1$ & $31 \cdot 8$ & $27 \cdot 4$ & 0.89 & 0.020 & $<0.001$ & 0.002 \\
\hline Total SFA & 244 & 266 & 259 & 252 & 258 & 253 & $6 \cdot 1$ & 0.018 & - & - \\
\hline Total MUFA & 266 & 285 & 285 & 266 & 277 & 274 & $7 \cdot 1$ & - & - & - \\
\hline Total $n-6$ & 41.6 & $40 \cdot 2$ & $32 \cdot 7$ & $49 \cdot 1$ & 41.2 & $40 \cdot 7$ & 0.85 & - & $<0.001$ & - \\
\hline Total $n-3^{*}$ & $48 \cdot 9$ & 41.9 & $24 \cdot 8$ & $66 \cdot 0$ & $48 \cdot 3$ & $42 \cdot 4$ & 1.27 & $<0.001$ & $<0.001$ & 0.003 \\
\hline Total PUFA & 90.5 & $82 \cdot 2$ & 57.5 & 115 & 89.5 & 83.1 & 1.90 & 0.005 & $<0.001$ & 0.024 \\
\hline
\end{tabular}

SEM, pooled standard error of mean: standard deviation/ $\sqrt{ } n$; L-C, L-carnitine; L, lipid; E, exercise; -, NS.

${ }^{*}$ Significant $(P<0.05)$ interaction between $\mathrm{L}$-carnitine and lipid.

determined by a series of systems that transport L-carnitine into cells against a concentration gradient, and the liver plays a unique role in the whole-body L-carnitine homeostasis. In the present study, the levels of dietary L-carnitine (15 and $1000 \mathrm{mg} / \mathrm{kg}$ ) were selected to represent the below and above optimal level for catfish ${ }^{(26,28)}$. L-Carnitine deficiency can be obtained by decreasing synthesis ${ }^{(5)}$, increasing excretion ${ }^{(29)}$ or by reducing dietary L-carnitine availability. In the present study, L-carnitine deficiency was induced by limiting dietary lysine level to a sub-optimum level, so as to restrict biosynthesis and reducing dietary L-carnitine availability ${ }^{(5)}$. Ozório $^{(30,31)}$ and Harpaz ${ }^{(4)}$ suggested that when limiting the level of L-carnitine precursor, L-carnitine supplementation might compensate for the low level of a dietary precursor. The optimal L-carnitine level seems to vary and depends on several biotic and abiotic factors, such as age, diet, metabolic conditions and tissue dependence on FA oxidation.

In the present study, free and acyl L-carnitine contents in muscle and plasma were linearly correlated with dietary L-carnitine level ( 3 to 5 times greater in the L-carnitine supplemented groups than in the non-supplemented groups). The ability to take up and retain dietary L-carnitine was previously observed in African catfish ${ }^{(17,26)}$, as well as in Atlantic

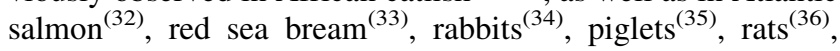
among others.

L-Carnitine concentrations are typically higher in tissues than in extracellular fluid compartments ${ }^{(5)}$. Indeed, in the present study the concentration of L-carnitine was over 50 times higher in skeletal muscle than that in plasma. Free and acyl L-carnitine content in skeletal muscle was linearly correlated $\left(R^{2} 0 \cdot 91\right)$ with plasma L-carnitine, indicating that L-carnitine supplements were taken up by tissues and available for intermediary metabolism. Since L-carnitine determination

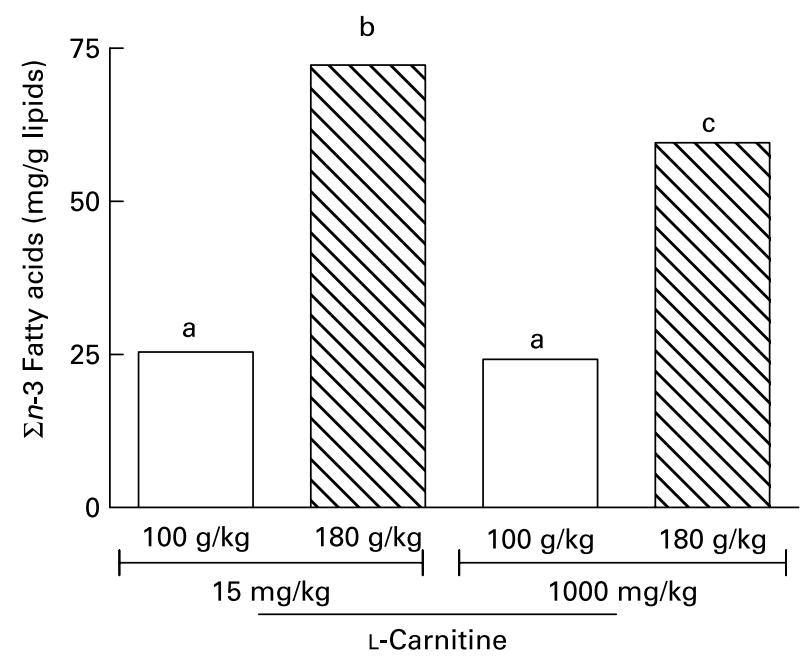

Fig. 3. Combined effects of dietary lipid $(100 \times 180 \mathrm{~g} / \mathrm{kg})$ and L-carnitine levels $\left(15 \times 1000 \mathrm{mg}\right.$ L-carnitine/kg diet) on liver total $n-3$ PUFA. ${ }^{\text {a,b,c }}$ Values with unlike letters were significantly different $(P<0.05)$. 


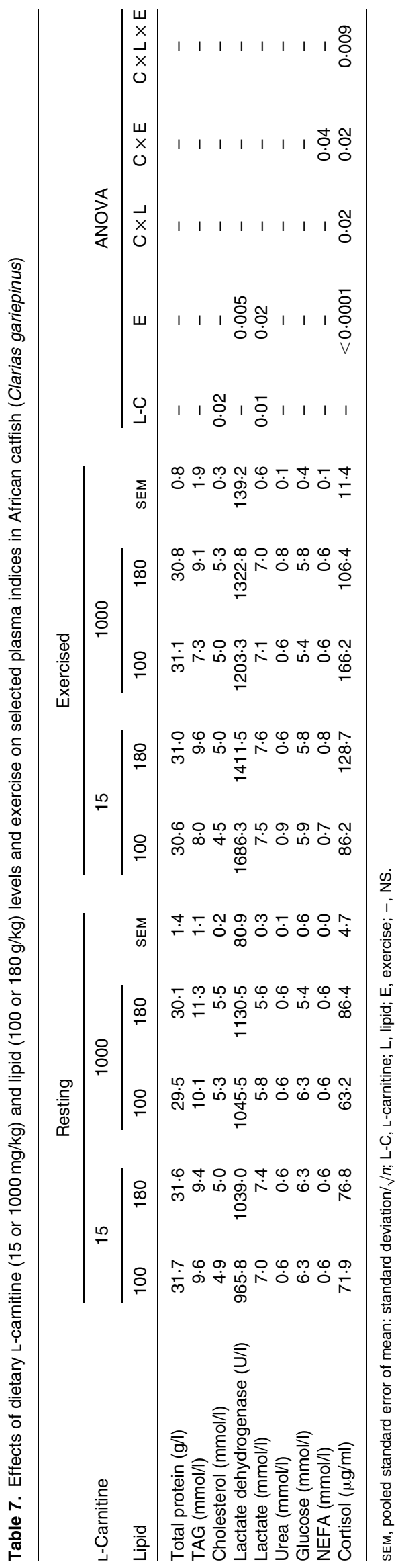

in plasma samples is a non-invasive method (favouring repeated measurements throughout the experimental period), the relation between plasma and muscle L-carnitine levels was validated from the results obtained from previous studies $^{(3,17,22,26)}$, and because muscle tissue is the main storage site of L-carnitine, it is plausible to infer that plasma L-carnitine content can be a good indicator of muscle L-carnitine content in African catfish.

Dietary lipid supplementation appears to elevate L-carnitine levels in the African catfish. Changes in optimal L-carnitine level are correlated with changes in the amount of circulating L-carnitine. The size of L-carnitine pool in animals is maintained by a combination of L-carnitine from supplemental sources, a modest rate of biosynthesis and an efficient reabsorption $^{(5)}$. In the present study, an increase in the dietary lipid level caused a $30 \%$ increase in muscle and in plasma L-carnitine content. In human subjects, plasma L-carnitine concentration is higher after a high-fat or high-protein $\operatorname{diet}^{(37)}$. As a consequence, more L-carnitine is reabsorbed, however, at lower efficiency rate. Berger \& Sachan ${ }^{(38)}$ observed that the efficiency of L-carnitine reabsorption in rats decreased with increasing levels of circulating L-carnitine, thus more L-carnitine is excreted. These observations suggest that dietary lipid levels may increase L-carnitine deposition, although body esterified L-carnitine level is often subestimated due to losses from excretion.

In fish, plasma and muscle L-carnitine contents increased with dietary L-carnitine level ${ }^{(3,22,39)}$, as has been also observed for other species ${ }^{(2,40)}$. Gaylord \& Gatlin ${ }^{(39)}$ showed that hybrid striped bass fed $3000 \mathrm{mg}$ L-carnitine $/ \mathrm{kg}$ diet, had differences ranged from 11 to $61 \mathrm{mg}$ L-carnitine/g muscle for fish fed 5 and $20 \%$ lipid, respectively. The mechanism by which this occurs is not clear, but it seems that L-carnitine transport and utilisation may be influenced by dietary lipid level.

Exercise significantly altered the free L-carnitine levels in muscle tissue. From rest to $3-\mathrm{h}$ of swimming at $1-\mathrm{BL} / \mathrm{s}$, muscle free L-carnitine content decreased from 6 to $20 \%$. Hiatt et al. ${ }^{(41)}$ observed in human subjects that from rest to $10 \mathrm{~min}$ of high-intensity exercise, free L-carnitine content decreased by $66 \%$. According to Hiatt et al. ${ }^{(41)}$, the alteration of the ratio esterified-to-free is dependent on the workload and is poorly reflected in the plasma and urine carnitine pools. In the present study, however, exercise did not affect the ratio esterified-to-free L-carnitine. Presumably, the workload applied to catfish was not very intense, and an extension of the swimming trial would have caused a more drastic alteration.

In the present study, the amount of lysine was $1.1 \%$, in comparison to the optimal $1.5 \%$ as determined for catfish ${ }^{(42)}$. Burtle \& Liu ${ }^{(43)}$ investigated the effects of $0.1 \%$ dietary L-carnitine in diets containing $1.1,1.4$ and $1.7 \%$ dietary lysine in channel catfish. L-Carnitine did not cause any effects on the growth rate, but a combination of L-Carnitine and lysine reduced body lipid content and visceral fat and increased body protein.

In the present study, L-Carnitine supplements had no clear effect on growth, but clearly affected the lipid and energy content in liver. The limited influence of L-Carnitine on African catfish growth and feed efficiency has been noted in a previous study in African catfish ${ }^{(26)}$, as well as in other fish species $^{(33,44,45)}$. 
Table 8. Effects of dietary L-carnitine (15 or $1000 \mathrm{mg} / \mathrm{kg}$ ) and lipid (100 or $180 \mathrm{~g} / \mathrm{kg}$ ) levels on respiratory gas exchange ( $\mathrm{mmol} / \mathrm{kg}$ per $\mathrm{h}$ ) and respiratory and nitrogen quotients in exercised African catfish (Clarias gariepinus)

\begin{tabular}{|c|c|c|c|c|c|c|c|c|}
\hline \multirow{2}{*}{$\begin{array}{l}\text { L-Carnitine } \\
\text { Lipid }\end{array}$} & \multicolumn{2}{|c|}{15} & \multicolumn{2}{|c|}{1000} & \multirow[b]{2}{*}{ SEM } & \multicolumn{3}{|c|}{ ANOVA } \\
\hline & 100 & 180 & 100 & 180 & & $\mathrm{~L}-\mathrm{C}$ & L & $C \times L$ \\
\hline $\mathrm{O}_{2}$ consumed $\left(\mathrm{O}_{2}\right)$ & $21 \cdot 3$ & $20 \cdot 3$ & $22 \cdot 0$ & $21 \cdot 0$ & 0.6 & - & - & - \\
\hline $\mathrm{CO}_{2}$ excretion $\left(\mathrm{CO}_{2}\right)$ & $13 \cdot 1$ & $14 \cdot 7$ & $14 \cdot 3$ & $13 \cdot 2$ & 0.6 & - & - & - \\
\hline $\mathrm{N}-\mathrm{NH}_{4}^{+}$excretion (N) & 1.03 & 1.06 & 1.63 & 0.93 & 0.08 & 0.01 & 0.01 & 0.05 \\
\hline$R Q$ & 0.62 & 0.72 & 0.65 & 0.63 & 0.01 & 0.03 & - & 0.03 \\
\hline Nitrogen quotient (NQ) & 0.07 & $0 \cdot 1$ & 0.12 & 0.08 & 0.02 & - & - & - \\
\hline
\end{tabular}

SEM, pooled standard error of mean: standard deviation $/ \sqrt{ } n$; L-C, L-carnitine; L, lipid; -, NS.

Some other studies have shown dramatic influences of dietary L-carnitine on body composition and on growth performance. Ji et al. ${ }^{(32)}$ noted that Atlantic salmon fed diets supplemented with L-carnitine had reduced fillet lipid. Chatzifotis et al. ${ }^{(46)}$ reported an increase in growth rate and feed efficiency in red sea bream when L-carnitine was supplemented at $1087 \mathrm{mg} / \mathrm{kg}$ diet. In a classical dose-response study, Torreele et al. ${ }^{(28)}$ clearly observed that dietary L-carnitine and lipid supplementation significantly affected performance of $5 \mathrm{~g}$ African catfish. In contrast, no significant effect on performance of African catfish juveniles growing from 9-32 g was observed in the present study. The difference in fish size might explain the different results obtained by Torreele et al. ${ }^{(28)}$ working in the same laboratory and with the same species as in the present study. During early life stages when fish are building up new tissues at very fast rate, a very high-energy demand has to be covered. Since endogenous L-carnitine synthesis is less developed in younger animals, dietary L-carnitine requirement might be more evident in $5 \mathrm{~g}$ than in $9 \mathrm{~g}$ African catfish.

In liver, DM, energy and lipid content increased, and protein decreased with increasing dietary lipid level. As a consequence, protein to lipid ratio also decreased. Since liver is the major site of lipid and glycogen deposition in fish, it is expected to observe a positive relationship between dietary lipid content and body lipid ${ }^{(47)}$. Dietary L-carnitine supplementation significantly reduced DM, energy and lipid content of the liver. The effects of dietary L-carnitine on proximate composition of several fish species have been inconsistent. Dietary L-carnitine did not alter tissue composition of rainbow trout ${ }^{(45)}$ or hybrid tilapia ${ }^{(48)}$. On the other hand, dietary L-carnitine reduced tissue lipid concentrations in rohu ${ }^{(49)}$, tilapia ${ }^{(50)}$, channel catfish $^{(43)}$, European sea bass $^{(51)}$ and Atlantic salmon ${ }^{(32)}$. Opposite to the latter studies, dietary L-carnitine increased lipid concentrations in liver and muscle of red sea bream ${ }^{(46)}$.

In teleost fish, the FA composition and content of muscle and liver is mainly dependent of the profile of dietary FA source. This effect might, however, be modulated by selective FA oxidation and modification of absorbed $\mathrm{FA}^{(52)}$. In the present experiment, increased fish oil incorporation in the diets resulted in an increased FA intake but had no major effects on FA profile of diets. Moreover, the intake of long-chain PUFA was quite high even with lower fat diets, so minimal endogenous modification of absorbed PUFA can be expected. Thus, in the present study, the FA oxidation might become the most important factor determining the FA composition of tissues. To our best knowledge, the selectivity mechanism regulating
FA oxidation in fish are poorly understood ${ }^{(52)}$, and our data suggest that increased FA intake may promote FA oxidation and both carnitine and exercise might influence the regulation of FA oxidation selectivity, as discussed later.

Supplementing high fish oil level did not change the total FA content of muscle, but changed the FA profile with increased PUFA coupled with decreased MUFA and SFA concentration. The increased FA intake might led to an increase FA oxidation, as observed in rainbow trout ${ }^{(53)}$ and in mice ${ }^{(54)}$, and because both SFA and MUFA are expected to be oxidised preferentially to PUFA $^{(52)}$, these will result in an altered FA composition of muscle.

In liver, the enrichment on both $n-6$ and $n-3$ PUFA induced by elevated fish oil level was also observed but was highest in animals fed non-supplemented L-carnitine diets. L-carnitine might facilitate the oxidation of $n-3$ PUFA, thus attenuating its accumulation in the liver. This is consistent with earlier studies on red sea bream, where is suggested that, L-carnitine supplementation affected FA metabolism by shifting the oxidation from $\mathrm{C} 14-\mathrm{C} 18$ to $\mathrm{C} 20-\mathrm{C} 22 \mathrm{FA}^{(46,55)}$.

Muscle of exercised fish had lower total FA content (mg/g lipid) and lower total lipids than the rested animals. This may indicate increased FA oxidation in muscles to couple with increased energy demand in exercised fish. In rested animals, $n-3$ PUFA are probably less oxidised than the other FA, but that selectivity mechanism regulating FA oxidation might be by-passed with increasing energy demand, such as in exercised fish. In that case, $n-3$ PUFA, SFA and MUFA are oxidised at similar rates.

Dietary L-carnitine levels did only influence cholesterol and lactate as plasma biochemical parameters. Exercise significantly elevated lactate and lactate dehydrogenase, which may indicate the activation of the anaerobic glycolysis to provide energy during exercise ${ }^{(56-58)}$. These findings are consistent with previous studies ${ }^{(59-64)}$. The nitrogen excretion can be divided in two different components: endogenous and exogenous (postprandial) excretion ${ }^{(65)}$. The endogenous excretion reflects the 'turn-over' body protein and the exogenous excretion, the result of the catabolism of dietary protein.

It is generally observed that the postprandial nitrogen excretion rates represent approximately $35-40 \%$ of the digestible nitrogen ingestion and may vary with the dietary protein to lipid ratio, the quality of the ingredients and the feed processing technique ${ }^{(66,67)}$. As demonstrated in previous studies with salmonids, ammonia excretion represents about $90 \%$ of the total excretion of the nitrogenous compounds ${ }^{(68-72)}$. The lowest values were observed in unfed fish, during which urea excretion represented $20 \%$ of the nitrogen excretion. 
Fuel oxidation preferences are often realised in changes of the RQ rates ${ }^{(73)}$. According to Kleiber ${ }^{(18)}, 1.0$ is the upper limit for aerobic catabolism and usually ranges between 0.65 and 1.0. The lower value indicates lipid oxidation and the higher value carbohydrate oxidation. In the present study, the RQ rates vary between 0.62 and 0.72 , and the pattern is in agreement with the variation observed in our previous study with African catfish. Ozório et al. ${ }^{(22)}$ reported a reduction in RQ in fish fed L-carnitine supplementation, particularly when dietary lipid level was high. In the present study, dietary L-carnitine supplementation significantly reduced RQ and nitrogen $\left(\mathrm{N}-\mathrm{NH}_{4}^{+}\right)$excretion in fish fed $180 \mathrm{~g} / \mathrm{kg}$. There were interaction effects between L-carnitine and lipid for RQ and nitrogen excretion, indicating that dietary L-carnitine effects on lipid, and indirectly on protein catabolism, may be conditioned by the dietary lipid level.

We assessed an experimental set up with isonitrogenous diets containing two lipid levels, two L-carnitine levels and a resting or a moderate swimming protocol $(1.5 \mathrm{BL} / \mathrm{s})$. Swimming activity causes fish to have less lipid reserves than those of sedentary fish. Plasma cortisol level was also lower in the resting fish when compared with exercised fish. Catfish is an air-breathing fish species and swimming does prevent this species from this additional source of oxygen supply because vertical migration is prevented and the swim tunnel is a closed system. However, the higher cortisol levels in the swimming group can be an advantageous condition because cortisol acts to mobilise fuel stores to maintain metabolic homeostasis and thus exerts effects on metabolism directly ${ }^{(74)}$. In addition, Milligan et al. ${ }^{(75)}$ observed that trout are able to recover much faster when they are allowed to swim at low speeds after collapse.

Swimming performance may change as a result of nutritional status ${ }^{(76)}$. The rate of cellular lipid oxidation is determined by the energy requirements of the working muscles and by the availability of free L-carnitine delivery to muscle mitochondria ${ }^{(17)}$. L-Carnitine may result in an elevated capacity for lipid oxidation in muscle mitochondria. During exercise, lipids oxidised in locomotory muscles can come from the circulation or from intramuscular reserves. Circulatory lipids and cholesterol are transported from the gut and from hepatic or adipose tissue stores ${ }^{(77)}$ by lipoproteins ${ }^{(78)}$.

The increase in concentrations of plasma lactate observed in the present study, normally associated with increased glycolytic processes, was correlated with exercise and is consistent with other studies ${ }^{(79-81)}$.

Little information is available about the release and timescale for excretion of lactic acid from the active tissues in circulation. In addition, lactic acid may not solely be considered as an end product causing fatigue and acid-base disturbances, but also may be considered as a substrate for oxidative tissues, such as red and cardiac muscle. In principle, using lactate as a substrate, hepatic gluconeogenesis (i.e. the Cori cycle) and in situ glyconeogenesis in white and red muscle may also occur ${ }^{(79)}$. Swimming has the advantage that it activates blood perfusion of most tissues, and thus allows rapid oxidisation of the accumulated lactate that quickly replenishes the $\mathrm{HCO}_{3}^{-}$pool ${ }^{(82)}$. The re-synthesis of lactate to glycogen will also help to restore metabolic acidosis. So, possibly slow swimming may be the natural way of recovery from a bout of exercise.

\section{Acknowledgements}

This research received no specific grant from any funding agency in the public, commercial or not-for-profit sectors. We declare that there are no conflicts of interest regarding the present paper. R. O. A. O., M. W. A. V., J. A. J. V. and E. A. H. designed the study. R. O. A. O. performed the animal experiments, analysed and interpreted the data. R. O. A. O. and V. J. T. V. G. designed the swimming experiment. R. J. B. B. analysed and interpreted the FA data. J. A. J. V. and E. A. H. supervised the experiments and provided administrative, technical and material support. R. O. A. O. wrote the draft of the manuscript. All the authors critically reviewed the manuscript.

\section{References}

1. Chatzifotis S (1998) The effect of dietary L-carnitine on growth and lipid composition of fish. PhD Dissertation, Tokyo University of Fisheries.

2. Heo KN, Odle J \& Han IK (2000) Effects of dietary L-carnitine and protein level on plasma carnitine, energy and carnitine balance, and carnitine biosynthesis of $20 \mathrm{~kg}$ pigs. Asian-Aust $J$ Anim Sci 13, 1568-1575.

3. Ozório ROA, Verreth JAJ, Aragão CR, et al. (2003) Dietary carnitine supplements increased lipid metabolism and decreased protein oxidation in African catfish (Clarias gariepinus) juveniles fed high fat levels. J Aquac Tropics 18, 225-238.

4. Harpaz S (2005) L-Carnitine and its attributed functions in fish culture and nutrition - a review. Aquaculture 249, 3-21.

5. Rebouche CJ \& Seim H (1998) Carnitine metabolism and its regulation in microorganisms and mammals. Annu Rev Nutr 18, 36-61.

6. Gulcin I (2006) Antioxidant and antiradical activities of L-carnitine. Life Sci 78, 803-811.

7. Heo K, Odle J, Han IK, et al. (2000) Dietary L-carnitine improves nitrogen utilization in growing pigs fed low energy, fat-containing diets. $J$ Nutr 130, 1809-1814.

8. Demmelmair H, Sauerwald T, Koletzko B, et al. (1997) New insights into lipid and fatty acid metabolism via stable isotopes. Eur J Pediatrics 156, S70-S74.

9. Gomes MD \& Tirapegui J (2000) Relation of some nutritional supplements and physical performance. Arch Latinoam Nutr 50, 317-329.

10. Janssens GPJ, Buyse J, Sweynaeve M, et al. (1998) The reduction of heat production in exercising pigeons after L-carnitine supplementation. Poult Sci 77, 578-584.

11. Kraemer WJ \& Volek JS (2000) L-Carnitine supplementation for the athlete, a new perspective. Ann Nutr Metab 44, 88-89.

12. Sachan DS \& Hongu $\mathrm{N}$ (2000) Increases in $\mathrm{VO}_{2 \max }$ and metabolic markers of fat oxidation by caffeine, carnitine, and choline supplementation in rats. $J$ Nutr Biochem 11, 521-526.

13. Souffleux G (1994) Benefit of using Rossovet carnitine for horses in preparation for amateur carriage-racing. Pratique Vétérinaire Équine 26, 241-248.

14. Spriet L, Perry C \& Talanian J (2008) Legal pre-event nutritional supplements to assist energy metabolism. Essays Biochem 44, 27-44.

15. Panjwani U, Thakur L, Anand JP, et al. (2007) Effect of L-carnitine supplementation on endurance exercise in normobaric/ normoxic and hypobaric/hypoxic conditions. Wilderness Environ Med 18, 169-176.

16. Tsakiris T, Angelogianni P, Tesseromatis C, et al. (2008) Effect of L-carnitine administration on the modulated rat brain protein concentration, acetylcholinesterase, $\mathrm{Na}^{+} \mathrm{K}^{+}$-ATPase and 
$\mathrm{Mg}^{2+}$-ATPase activities induced by forced swimming. Br J Sports Med 42, 367-372.

17. Ozório R, Van Ginneken V, Van den Thillart G, et al. (2005) Dietary L-carnitine maintains energy reserves and delays fatigue of exercised African catfish fed high fat diets. Sci Agric 62, 208-213.

18. Kleiber M (1987) The Fire of Life. An Introduction to Animal Energetics. Malabar, FL: Robert E. Krieger Publ. Co..

19. Brouwer E (1958) On simple formulae for calculating the heat expenditure and the quantities of carbohydrate and fat metabolized in ruminants, from data on gaseous exchange and urine-N. In I Symposium on Energy Metabolism, pp. 182-194. Rome: European Association for Animal Production.

20. Lauff RF \& Wood CM (1997) Effects of training on respiratory gas exchange, nitrogenous waste excretion, and fuel usage during aerobic swimming in juvenile rainbow trout (Oncorhynchus mykiss). Can J Fish Aquat Sci 54, 566-571.

21. Kutty MN (1968) Respiratory quotients in goldfish and rainbow trout. J Fish Res Bd Can 25, 1689-1728.

22. Ozório ROA, van Eekeren THB, Huisman EA, et al. (2001) Effects of dietary carnitine and protein energy: nonprotein energy ratios on growth, ammonia excretion and respiratory quotient in African catfish, Clarias gariepinus (Burchell) juveniles. Aquac Res 32, 406-414.

23. Ali MZ \& Jauncey K (2004) Optimal dietary carbohydrate to lipid ratio in African catfish Clarias gariepinus (Burchell 1822). Aquac Int 12, 169-180.

24. Van Ginneken VJT (2006) Simulated migration of European Eel (Anguilla anguilla, Linnaeus 1758). PhD dissertation no. 3979, Wageningen University, The Netherlands, pp. 309.

25. Christiansen RZ \& Bremer J (1978) Acetylation of Tris(hydroxymethyl)aminomethane (Tris) and Tris derivatives by carnitine acetyltransferase. FEBS Lett 86, 99-102.

26. Ozório ROA, Uktoseja JLA, Huisman EA, et al. (2001) Changes in fatty acid concentrations in tissues of African catfish, Clarias gariepinus Burchell, as a consequence of dietary carnitine, fat and lysine supplementation. $B r J$ Nutr 86, $623-636$.

27. Ruane NM, Huisman EA \& Komen J (2001) Plasma cortisol and metabolite level profiles in two isogenic strains of common carp during confinement. J Fish Biol 59, 1-12.

28. Torreele E, Vandersluiszen A \& Verreth J (1993) The effect of dietary L-carnitine on the growth-performance in fingerlings of the African catfish (Clarias gariepinus) in relation to dietarylipid. Br J Nutr 69, 289-299.

29. Nelson HK, Lauber RP \& Sheard NF (2001) Effect of various levels of supplementation with sodium pivalate on tissue carnitine concentrations and urinary excretion of carnitine in the rat. $J$ Nutr Biochem 12, 242-250.

30. Ozório ROA (2001) Dietary L-carnitine and energy and lipid metabolism in African catfish (Clarias gariepinus) juveniles. $\mathrm{PhD}$ dissertation no. 3092, Wageningen University, Holland.

31. Ozório ROA (2009) Dietary L-carnitine supplementation to cultivated fish: a mini-review. Curr Nutr Food Sci 5, 40-48.

32. Ji H, Bradley TM \& Tremblay GC (1996) Atlantic salmon (Salmo salar) fed L-carnitine exhibit altered intermediary metabolism and reduced tissue lipid, but no change in growth rate. J Nutr 126, 1937-1950.

33. Chatzifotis S, Takeuchi T, Watanabe T, et al. (1997) The effect of dietary carnitine supplementation on growth of rainbow trout fingerlings. Fish Sci 63, 321-322.

34. Bell FP, Vidmar TJ \& Raymond TL (1992) L-Carnitine administration and withdrawal affect plasma and hepatic carnitine concentrations, plasma lipid and lipoprotein composition, and in vitro hepatic lipogenesis from labeled mevalonate and oleate in normal rabbits. J Nutr 122, 959-966.
35. Baltzell JK, Bazer FW, Miguel SG, et al. (1987) The neonatal piglet as a model for human neonatal carnitine metabolism. J Nutr 117, 754-757.

36. Tsai AC, Romsos DR \& Leveille GA (1974) Significance of dietary carnitine for growth and carnitine turnover in rats. J Nutr 104, 782-792.

37. Stadler DD, Catherine AC \& Rebouche CJ (1993) Effect of dietary macronutrient content on carnitine reabsorption. Am J Clin Nutr 58, 868-872.

38. Berger R \& Sachan DS (1991) Effects of supplementary levels of L-carnitine on blood and urinary carnitines and on the portal-systemic blood-ethanol concentrations in the rat. $J$ Nutr Biochem 2, 382-386.

39. Gaylord TG \& Gatlin DM (2000) Dietary lipid level but not L-carnitine affects growth performance of hybrid striped bass (Morone chrysops $\times$ M. saxatilis). Aquaculture 190, 237-246.

40. Greenwood RH, Titgemeyer EC, Stokka GL, et al. (2001) Effects of L-carnitine on nitrogen retention and blood metabolites of growing steers and performance of finishing steers. J Anim Sci 79, 254-260.

41. Hiatt WR, Regensteiner JG, Wolfel EE, et al. (1989) Carnitine and acylcarnitine metabolism during exercise in humans dependence on skeletal-muscle metabolic state. J Clin Invest 84, 1167-1173.

42. Robinson EH, Wilson RP \& Poe WE (1980) Re-evaluation of the lysine requirement and lysine utilization by fingerling channel catfish. $J$ Nutr 110, 2313-2316.

43. Burtle GJ \& Liu Q (1994) Dietary carnitine and lysine affect channel catfish lipid and protein composition. $J$ World Aquac Soc 25, 169-174.

44. Chatzifotis S \& Takeuchi T (1997) Effect of supplemental carnitine on body weight loss, proximate and lipid compositions and carnitine content of red sea bream (Pagrus major) during starvation. Aquaculture 158, 129-140.

45. Rodehutscord M (1995) Effects of supplemental dietary L-carnitine on the growth and body composition of rainbow trout (Oncorhynchus mykiss) fed high-fat diets. J Anim Physiol Anim Nutr 73, 276-279.

46. Chatzifotis S, Takeuchi T \& Seikai T (1995) The effect of dietary L-carnitine on growth performance and lipid composition in Red sea bream fingerlings. Fish Sci 61, 1004-1008.

47. Jobling M (2001) Nutrient partitioning and the influence of feed composition on body composition. In Food Intake in Fish, pp. 354-375 [D Houlihan, T Boujard and M Jobling, editors]. Oxford: Blackwell Science.

48. Becker K, Schreiber S, Angoni C, et al. (1999) Growth performance and feed utilization response of Oreochromis niloticus $\times$ Oreochromis aureus hybrids to L-carnitine measured over a full fattening cycle under commercial conditions. Aquaculture 174, 313-322.

49. Keshavanath P \& Renuka P (1998) Effect of dietary L-carnitine on growth and body composition of fingerling rohu, Labeo rohita (Hamilton). Aquac Nutr 4, 83-87.

50. Jayaprakas V, Sambhu C \& Sunil Kumar S (1996) Effect of dietary L-carnitine on growth and reproductive performance of male Oreochromis mossambicus (Peters). Fish Technol 33, 84-90.

51. Santulli A \& D'Amelio V (1986) Effect of supplemental dietary carnitine on the growth and lipid metabolism of hatchery-reared sea bass, Dicentrarchus labrax L.. Aquaculture 59, 177-186.

52. Tocher DR (2003) Metabolism and functions of lipids and fatty acids in Teleost Fish. Rev Fish Sci 11, 107-184.

53. Kolditz C, Borthaire M, Richard N, et al. (2008) Liver and muscle metabolic changes induced by dietary energy content and genetic selection in rainbow trout (Oncorhynchus mykiss). Am J Physiol Regul Integr Comp Physiol 294, R1154-R1164. 
54. Turner N, Bruce CR, Beale SM, et al. (2007) Excess lipid availability increases mitochondrial fatty acid oxidative capacity in muscle: evidence against a role for reduced fatty acid oxidation in lipid-induced insulin resistance in rodents. Diabetes 56, 2085-2092.

55. Chatzifotis S, Takeuchi T \& Seikai T (1996) The effect of dietary carnitine supplementation on growth of red sea bream (Pagrus major) fingerlings at two levels of dietary lysine. Aquaculture 147, 235-248.

56. Dobson GP, Parkhouse WS \& Hochachka PW (1987) Regulation of anaerobic atp-generating pathways in trout fast-twitch skeletal-muscle. Am J Physiol 253, R186-R194.

57. Driedzic WR \& Hochachka PW (1976) Control of energymetabolism in fish white muscle. Am J Physiol 230, 579-582.

58. Mommsen TP \& Hochachka PW (1988) The purine nucleotide cycle as 2 temporally separated metabolic units - a study on trout muscle. Metab Clin Exp 37, 552-556.

59. Butler PJ, Metcalfe JD \& Ginley SA (1986) Plasma catecholamine in the lesser spotted dogfish and rainbow trout at rest and during different levels of exercise. J Exp Biol 123, 409-421.

60. Johnston IA \& Goldspink G (1973) A study of the swimming performance of crucian carp (Carassius carassius L.) in relation to the effects of exercise and recovery on the biochemical changes in myotomal muscles and liver. J Fish Biol 5, 249-260.

61. Driedzic WR \& Kiceniuk JW (1976) Blood lactate levels in free-swimming rainbow trout (Salmo gairdneri) before and after strenuous exercise resulting in fatigue. $J$ Fish Res $B d$ Can 33, 173-176.

62. Wokoma A \& Johnston IA (1981) Lactate production at high sustainable cruising speeds in rainbow trout (Salmo gairdneri Richardson). J Exp Biol 90, 361-364.

63. Duthie G (1982) The respiratory metabolism of temperature adapted flatfish at rest and during swimming activity and the use of anaerobic metabolism at moderate swimming speeds. J Exp Biol 97, 359-373.

64. Boutilier RG, Heming TA \& Iwama GK (1984) Fish physiology. In Physicochemical Parameters for Use in Fish Respiratory Physiology, pp. 403-430 [WS Hoar and DJ Randall, editors]. New York: Academic Press.

65. Forsberg OI (1997) The impact of varying feeding regimes on oxygen consumption and excretion of carbon dioxide and nitrogen in post-smolt Atlantic Salmon, Salmo salar L.. Aquac Res 28, 29-41.

66. Kaushik SJ \& Cowey CB (1991) Ammoniogenesis and dietary factors affecting nitrogen excretion. In Nutritional Strategies and Aquaculture Waste, pp. 3-19 [CB Cowey and CY Cho, editors]. Guelph: University of Guelph.

67. Watanabe T (1991) Past and present approaches to aquaculture waste managements in Japan. In Nutrient Strategies and Aquaculture Waste. Proceedings of the First International Symposium on Nutrient Strategies in Management of Aquaculture Waste, pp. 137-154 [CB Cowey and CY Cho, editors]. Ontario: University of Guelph.

68. Beamish FWH \& Thomas E (1984) Effects of dietary protein and lipid on nitrogen losses in rainbow trout (Salmo gairdneri). Aquaculture 41, 359-371.
69. Brett JR \& Zala CA (1975) Daily pattern of nitrogen excretion and oxygen consumption of sockeye salmon Oncorhynchus nerka under controlled conditions. J Fish Res Bd Can 32, 2479-2486.

70. Fivelstad S, Thomassen JM, Smith MJ, et al. (1990) Metabolite production rates from Atlantic salmon (Salmo salar L.) and Arctic char (Salvelinus alpinus L.) reared in single pass landbased brackish water and sea-water systems. Aquac Eng 9, $1-21$.

71. Médale F, Brauge C, Vallée F, et al. (1995) Effects of dietary protein/energy ratio, ration size, dietary energy source and water temperature on nitrogen excretion in rainbow trout. Water Sci Technol 31, 185-194.

72. Wiggs AJ, Henderson EB, Saunders RL, et al. (1989) Activity, respiration, and excretion of ammonia by Atlantic salmon (Salmo salar) smolt and postmolt. Can J Fish Aquat Sci 46, 790-795.

73. Xu D, Dhillon AS, Drake PG, et al. (1996) The transition from lipid to carbohydrate oxidation in response to re-feeding after starvation. Nutr Res 16, 1545-1553.

74. Van der Boon J, Van den Thillart GEEJM \& Addink ADF (1991) The effects of cortisol administration on intermediary metabolism in teleost fish. Comp Biochem Physiol A 100, $47-53$.

75. Milligan CL, Hooke GB \& Johnson C (2000) Sustained swimming at low velocity following a bout of exhaustive exercise enhances metabolic recovery in rainbow trout. $J$ Exp Biol 203, 921-926.

76. Martinez M, Guderley H, Dutil J-D, et al. (2003) Condition, prolonged swimming performance and muscle metabolic capacities of cod Gadus morhua. J Exp Biol 206, 503-5111.

77. Weber J-M \& Haman F (1996) Pathways for metabolic fuels and oxygen in high performance fish. Comp Biochem Physiol A 113, 33-38.

78. Salway JG (2006) Medical Biochemistry at A Glance, 2nd ed. Oxford: Blackwell Publishing Ltd.

79. Van Ginneken V, Boot R, Murk T, et al. (2004) Blood plasma substrates and muscle lactic-acid response after exhaustive exercise in common carp and trout: indications for a limited lactateshuttle. Anim Biol 54, 119-130.

80. Van Ginneken VJT, Balm P, Sommandas V, et al. (2002) Acute stress syndrome of the yellow European eel (Anguilla anguilla Linnaeus) when exposed to a graded swimming-load. Neth J Zool 52, 29-42.

81. Wood CM, Walsh PJ, Thomas S, et al. (1990) Control of red blood cell metabolism in rainbow trout after exhaustive exercise. J Exp Biol 154, 491-507.

82. Van Ginneken V, Coldenhoff K \& Boot R (2008) Depletion of high energy phosphates implicates post-exercise mortality in carp and trout: an in vivo 31P-NMR study. Comp Biochem Physiol A 149, 98-108.

83. Goelema JO, Spreeuwenberg MAM, Hof G, et al. (1998) Effect of pressure toasting on the rumen degradability and intestinal degradability of whole and broken peas, lupins and faba beans and a mixture of these feedstuffs. Anim Feed Sci Technol 76, $35-50$. 